\title{
L'allaitement maternel en Haïti comme rite de passage au carrefour des épistémologies du Nord et du Sud
}

\author{
Obrillant Damus \\ Enseignant-chercheur aux Universités d'État d'Haïti et Quisqueya
}

Revue Éducation, Santé, Sociétés Vol. 6, No 1 | pp. 57-74

Reçu le : 18/10/2019 | Accepté le : 28/12/2019

Résumé : Bien qu'il puisse être regardé à travers le prisme d'une théorie, l'allaitement humain est l'objet d'une réflexion multiréférentielle. Qu'il soit artificiel ou naturel, l'allaitement n'est pas un simple acte nourricier. Ancré dans une pensée aux dimensions multiples (symbolique, médicale, religieuse, mythologique et magique), il prend sens dans les creusets culturels et sociaux et constitue donc, de par ses variations anthropologiques, un phénomène complexe. Néanmoins, il existe des règles, des représentations et des mythes communs aux rites d'allaitement dans chaque communauté humaine. L'allaitement s'apparente à un rite de passage classique dont le vécu et les significations se situent au carrefour de l'universel et du particulier. II n'est pas un phénomène exclusivement biologique. La connaissance de l'invariant anthropologique que représente le rite d'allaitement ne peut se réaliser qu'à travers l'observation de celui-ci dans les différentes cultures humaines. Quelles sont les pratiques d'allaitement dans les communautés rurales d'Haïti? Quels sont les acteurs communautaires qui accomplissent des actions rituelles au profit des femmes allaitantes dans les milieux ruraux? Quels sont les facteurs culturels (mythes, tabous, valeurs, symboles, cosmovisions, etc.) qui les accompagnent? Dans quelle mesure la théorie des rites de passage de Van Gennep peut-elle contribuer à la compréhension du phénomène d'allaitement en milieu rural d'Haïti ?

Mots-clés : allaitement, rite de passage, nourrisson, femme allaitante.

\section{Introduction}

« Haïti » vient du mot indien Ayiti, « terre montagneuse, terre haute ». Dèyè mòn gen mòn (« II y a d'autres montagnes derrière une montagne »), dit un proverbe créole. Haïti, dans les Grandes Antilles, compte une population d'environ 11591279 habitants $^{1}$, dont 80 à $85 \%$ d'ascendance africaine et 15 à $20 \%$ issus du métissage ou d'origine européenne. On y parle deux langues officielles (le français depuis l'indépendance en 1804, et le créole, langue co-officielle à partir de 1987) et on y pratique la religion des Témoins de Jéhovah, la religion catholique, la religion protestante et le vodou. Le 12 janvier 2010, Haïti

1. Atlas des populations et pays du monde. 
fut victime d'un tremblement de terre qui a fait des milliers de morts et de sans-abris. Les ressources institutionnelles et l'État-Providence sont faibles. La famille-providence, le voisinage-providence et la communauté-providence jouent un rôle de premier plan dans la survie des populations rurales matériellement, médicalement et économiquement démunies $^{2}$. D'une manière générale, les femmes rurales accouchent à la maison pour des raisons d'ordre matériel (les centres de maternité sont rares et ne sont pas matériellement équipés dans les communautés rurales), économique et culturel (Damus, 2012). En milieu rural, l'allaitement maternel est une norme. D'après une enquête nationale ${ }^{3}:$ «a quasi-totalité des enfants sont allaités (97\%), mais seulement $47 \%$ d'entre eux sont mis au sein dans l'heure qui suit la naissance. Seulement $40 \%$ des enfants ont été allaités exclusivement au sein jusqu'à l'âge de 6 mois »(2013, p. 163).

Les pratiques d'allaitement s'observent dans toutes les sociétés humaines. Elles contribuent à la survie de ces dernières et participent à la lutte contre la vulnérabilité de la mère et de son nourrisson (Damus, 2016). Les rites d'allaitement maternel contribuent non seulement au maintien de la santé de ce dernier, mais encore à la lutte contre la pauvreté économique de nombreuses familles tant paysannes qu'urbaines dans les pays en voie de développement. De plus, la pratique de l'allaitement maternel joue un rôle non négligeable dans le développement durable (Damus, 2017; Bitoun, 1994 ; Radford, 1992), ou dans la conservation de la nature grâce à la transmission des savoirs, savoir-faire, symboles, normes, règles, interdits, représentations, croyances et des valeurs - qui sous-tendent la pérennité de ce phénomène - de génération en génération. Qu'il soit artificiel ou naturel, l'allaitement n'est pas un simple acte nourricier. Ancré dans une pensée aux dimensions multiples - symbolique, médicale, religieuse, mythologique et magique -, il prend sens dans les creusets culturels et sociaux et constitue, de par ses variations anthropologiques, un phénomène complexe (Desclaux et Taverne, 2000 ; Erny, 1988). Ses représentations varient d'une époque à l'autre (Dasen, 2015; Thirion, 2010 ; Lett et Morel, 2006 ; Rollet, 2005), d'une femme à l'autre (Badinter, 2010), d'une classe sociale à l'autre, d'une société à l'autre (Del Valle, 2000 ; Bartoli, 1998), voire au sein d'une même culture. II n'en demeure pas moins qu'il existe, dans chaque communauté humaine, des règles, des représentations et des mythes communs aux rites d'allaitement.

L'allaitement s'apparente à un rite de passage classique (Van Gennep, 1981) dont le vécu et les significations se situent au carrefour de l'universel et du particulier. La connaissance de l'invariant anthropologique que représente le rite d'allaitement ne peut se réaliser qu'à travers l'observation de celui-ci dans les différentes cultures humaines. Les femmes qui rencontrent des problèmes de lactation bénéficient de savoirs aux dimensions multiples (symbolique, magique, rationnelle, technique, masculine, féminine, communautaire ou collective) sur l'allaitement maternel. Quels sont les acteurs communautaires qui accomplissent des actions rituelles au profit des femmes allaitantes dans les milieux ruraux? Dans quelle mesure la théorie des rites de passage de Van Gennep peut-elle contribuer à la compréhension du phénomène d'allaitement? Comment les rites d'allaitement maternel

2. La solidarité vicinaire (des voisins et des voisines) et la solidarité familiale compensent, dans une large mesure, le manque ou l'absence de ressources extrafamiliales formelles dans les milieux ruraux (Damus, 2019).

3. Enquête Mortalité, Morbidité et Utilisation des Services [EMMUS-V]) par questionnaire commanditée par le Ministère de la Santé publique et de la Population (MSPP) et effectuée de janvier à juin 2012 par l'Institut Haïtien de l'Enfance auprès de plusieurs milliers de ménages $(n=13.227)$. 
font-ils passer les femmes et leur nourrisson d'un statut à un autre? En quoi l'allaitement maternel représente-t-il un rite de passage entre une mère et son enfant?

\section{Aspects méthodologiques}

Nous avons réalisé une enquête par entretiens directifs et observation auprès d'une quarantaine de femmes rurales illettrées et non-salariées, à l'exception de l'une d'entre elles qui exerce le métier d'institutrice. En janvier 2016, nous avons rencontré 20 informatrices dans trois petites communautés rurales contiguës qui sont administrativement rattachées à la ville de Saint-Marc (département de l'Ouest) : Kapyè, Pika, Jilbè. En février 2016, 20 autres mères ont été interviewées à Demontrèy, une communauté rurale dépendant administrativement de la ville de Jacmel (département du Sud-Est). L'objectif de cette recherche qualitative était d'amener ces femmes à décrire leurs pratiques d'allaitement dans leur langue maternelle (le créole haïtien). Lors du déroulement des entretiens de recherche, nous n'avons pas hésité à nous intéresser à l'histoire de vie de ces mères, ainsi qu'à des descriptions socio-économiques. Les entretiens, d'une durée moyenne de 70 minutes, ont été retranscrits intégralement. Pour les analyser de façon longitudinale et transversale, nous avons utilisé la méthode d'analyse de contenu de Bardin (1993). Plusieurs catégories ont été dégagées du matériel empirique. Ce sont des abstractions inductives qui condensent des récurrences ou des saillances sémantiques observées dans les discours des mères rurales. En voici quelques exemples parmi tant d'autres : le syndrome d'insuffisance lactée, les rites de stimulation de la sécrétion lactée (rites de stimulation de l'activité sécrétoire du sein) et de la descente du lait, les rites de purification de l'accouchée, les rites sympathiques et les rites alimentaires anti-hypogalactiques ou hypergalactiques.

\section{Contexte théorique et positionnement épistémologique}

Nous avons essayé de regarder l'allaitement maternel à travers le prisme de la théorie des rites de passage de Van Gennep (1981). Le vécu de l'allaitement se caractérise par trois moments, à savoir : la séparation, la marge et l'agrégation, auxquels sont associés des sentiments, des cognitions et des conduites sui generis. L'anthropologue Arnold Van Gennep a inventé ce schéma ternaire, dont la portée méthodologique et théorique est universelle, en vue de décrire et d'expliquer des rites de passage comme la naissance et le mariage. Ce schéma permet « d'ordonner et de rendre intelligibles des faits en apparence dispersés et incohérents »(Erny, 1994). Pour compenser les limites de l'apport de cette théorie à la compréhension de l'aspect psychologique et anthropologique de l'allaitement maternel, nous avons recouru à plusieurs travaux francophones et anglophones dont la théorie sociale cognitive de l'orientation de Robert W. Lent, Steven D. Brown et Gail Hackett (1994), la théorie de l'adaptation de Rudolf Moos (1987) et la théorie sociale cognitive d'Albert Bandura (1986).

Notre démarche épistémologique est plurielle. La pratique de l'allaitement maternel se situe au carrefour de l'épistémologie du Nord et des épistémologies du Sud, dont les « deux piliers sont " l'écologie des savoirs" et la "traduction interculturelle" (Santos, 2016). Les divers travaux développés dans l'ouvrage-programme « épistémologies du Sud » de Santos constituent, de notre point de vue, un nouveau cadre épistémologique pour les sciences humaines et sociales. 


\section{Le rôle des tradipraticiens de médecine créole dans l'allaitement}

L'approche du manque ou de la disparition du lait maternel est traversée de conceptions nomologico-causales (l'insuffisance ou la disparition du lait est perçue comme un phénomène procédant de lois naturelles. Dans ce cas, on fait appel à une matrone vulgairement appelée fanm chay, ou à un médecin-feuille) et de conceptions exocausales (là, le manque ou le tarissement du lait est considéré comme résultant d'un mauvais esprit, d'un loa ${ }^{4}$ ou sorcier. Dans ce cas, il faut consulter un houngan ou une mambo. Si après le troisième jour le lait ne « descend » pas dans le sein et qu'on relie ce problème à une attaque sorcellaire, c'est un houngan ou une mambo qui fabriquera les rites de stimulation de la sécrétion lactée). Deux types de rites sont réalisés par les matrones (sages-femmes traditionnelles), les médecins-feuilles, les houngans et les mambos au bénéfice des femmes qui viennent d'enfanter: les rites de stimulation de l'activité sécrétoire du sein d'une part et les rites de purification de l'accouchée d'autre part, cette dernière étant considérée comme une femme impure, souillée par le sang de l'accouchement. Ces tradipraticiens préparent des tisanes, des décoctions, des bains de feuilles et des massages. Ils connaissent non seulement les plantes qui favorisent la descente (fèy lete), ou le retour du lait (fèy letwon) dans les seins de la mère, mais encore celles qui purifient celle-ci. Les infusions de feuilles absorbées ont une propriété purificatrice dans la mesure où elles chassent les « sangs coagulés impurs »du ventre de l'accouchée. Après l'accouchement de son enfant, la femme est soumise à un ensemble de thérapies purificatrices. L'accouchée boit du thé de lachòy et d'asòsi (assorossi) pour se purifier.

\subsection{Les matrones}

Les matrones jouent un rôle important dans l'attribution des soins aux femmes enceintes, aux femmes allaitantes et aux nouveau-nés (Pourchez, 2011; Damus, 2012). En 2013, elles ont réalisé la plus grande proportion des accouchements non institutionnels : "Pour l'ensemble des départements, un total de 125.530 accouchements a été enregistré au cours de l'année 2013 avec 49,96\% réalisés au niveau institutionnel et 50,04\% au niveau non institutionnel. Au niveau institutionnel les accouchements normaux représentent $88,23 \%$ et ceux par césarienne 10,89\%. Les matrones ont réalisé $97,10 \%$ des accouchements non institutionnels. À noter que les départements de l'Ouest, de l'Artibonite et du Centre ont réalisé le plus grand nombre d'accouchements au cours de l'année soit respectivement $25,58 \%, 15,24 \%$ et 14,84 \% (Enquête Mortalité, Morbidité et Utilisation des Services [EMMUS-V]). Le mot matrone vient du latin, matrona, de mater (mère) avec un suffixe augmentatif, selon une dérivation similaire à pater (père), patron. La matrone est surtout valorisée là où il n'y a pas de formation médicale pour les femmes qui désirent apprendre formellement le métier d'accoucheuse. Une matrone, selon nos données empiriques, est une personne qui n'a pas de profil médical pour exercer légalement sa profession. La matrone est-elle vraiment « une accoucheuse qui exerce illégalement sa profession »? Nous pensons que c'est une personne qui est acceptée et valorisée par les membres de sa communauté rurale d'appartenance. Si le mot matrone désignait une femme sage, celui de sage-femme (mot composé) serait le résultat du processus de synthémisation des mots femme et sage. L'antéposition de l'adjectif sage au mot femme (sage-femme) semble être

4. Divinité du vodou haïtien (esprit, mystère, invisible). 
liée à un effet d'emphase : il s'agirait d'abord d'insister sur les vertus de l'accoucheuse traditionnelle. Le mot matrone existe en créole (matwòn). Mais c'est son synonyme « fanm chay » qui est le plus utilisé. On ne doit pas traduire le terme créole « fanm chay » par sage-femme. Le calque n'est pas toujours un bon procédé de traduction. Contrairement aux matrones, la sage-femme, selon l'Organisation mondiale de la santé (OMS), est « une personne qui a suivi un programme de formation reconnu dans son pays, a réussi avec succès les études afférentes et acquis les qualifications nécessaires pour être reconnue ou licenciée en tant que sage-femme ».

En français de France, le terme de matrone a acquis une acception péjorative. En Haïti, il s'emploie dans un sens mélioratif. Le terme créole «fanm chaj » doit être traduit par matrone ou sage-femme traditionnelle. La «fanm-chay » (femme de peine [sens imagé]) est une personne qui se met dans la peau de la femme en travail afin de l'aider à faire face à ses souffrances émotionnelles, physiques et psychologiques éphémères. Elles sont des illettrées-savantes. La plupart d'entre elles exercent aussi le métier de mambo et de médecin-feuille. Les matrones permettent aux femmes de leur communauté de développer des savoirs expérientiels sur la naissance et sur l'allaitement en les incitant à participer activement au processus de l'accouchement et à allaiter leur nourrisson.

La plupart des matrones, notamment celles que nous avons rencontrées à Jean-Rabel en 2012 (Damus, 2015) et en 2016, conseillent aux accouchées d'allaiter au sein leur bébé. Julie Pierre, une matrone jean-rabellienne, fait l'éloge de l'allaitement maternel en ces termes:

«L'enfant trouve tout ce qu'il lui faut (comme aliment) dans le lait maternel. Il n'a pas d'autre nourriture que ce dernier. Je suggère à la mère de ne pas acheter de lait pour nourrir le bébé. Elle doit utiliser son argent pour se soigner. Pourquoi? Le lait maternel contient tout ce dont a besoin l'enfant. II a des vitamines, des protéines, de l'eau, du sel, etc. C'est la raison pour laquelle, l'enfant doit être mis au sein à la naissance. Après avoir tété la mère, l'enfant pousse ses premiers excréments. Le méconium fait mal à celui-ci. Le lait de la mère s'est substitué au lòk. »

Pour préparer un «lòk», il faut utiliser des éléments tels que l'ail, I'huile de palma-christi, la noix muscade... Certaines matrones ont recours au lòk pour dégorger l'intestin des bébés après leur naissance. Cette pratique ancestrale a été abandonnée par de nombreuses matrones qui ont suivi une formation occidentalocentrée sur l'accouchement. Les pratiques occidentales d'intervention à grande vitesse en Haïti et ailleurs font disparaître beaucoup de savoirs locaux. C'est ce qu'on appelle un épistémicide pour reprendre un concept du sociologue portugais Boaventura De Sousa Santos (2016).

\subsection{Les médecins-feuilles}

Les personnes utilisant des plantes pour opérer des guérisons sont appelées médecinsfeuilles ou docteurs-feuilles. Elles n'ont pas besoin forcément d'être possédés par un esprit (loa) pour soigner. Le mot médecin-feuille (ou docteur-feuille) est une composition qui résulte de la juxtaposition linéaire de deux éléments basiques : médecin et feuille. Un médecin-feuille est un guérisseur qui utilise des plantes (et/ou des prières) pour soigner 
(phytothérapeute traditionnel) les autres. Les médecins-feuilles n'utilisent pas de loasguérisseurs (esprits) pour guérir des maladies, à la différence des mambos ou des houngans. Une femme enceinte qui a des douleurs abdominales peut consulter un médecin-feuille. Si une femme manque de lait, si sa complication mammaire est reliée à une étiologie endogène ou à un événement traumatique, elle pourra également consulter ce dernier. Pauline, mère de six enfants, nous explique comment un médecin-feuille avait facilité le retour de son lait quand il était « parti ${ }^{5}$ »:

«À la suite d'une grande émotion, le lait peut se tarir. II faut prendre des remèdes pour provoquer son retour. La mort d'un enfant de 16 mois avait fait partir mon lait. J'étais devenue "blanche". Ma tête était l'objet de plusieurs bains de feuilles. Ne vois-tu pas que mes cheveux sont devenus blancs? On avait mis trop de choses sur ma tête : des feuilles (fèy lèt, fèy manman lèt... ), de la cassave, du hareng saur, de l'écorce du grain de café préalablement portées à ébullition ou exposées au soleil. Ce traitement avait permis le retour du lait. »

\subsection{Les houngans et les mambos}

Un houngan est un prêtre du vodou qui, pour guérir des malades, doit être possédé par un esprit, communément appelé «loa ». II utilise des plantes médicinales et des produits ethnopharmaceutiques. Le houngan est «l'homme à qui on a recours dans toutes les circonstances de la vie, et qui sait se faire entendre des esprits. Chef d'une confrérie, il n'a de compte à rendre à personne, et pour chacun de ses clients, il représente la toutepuissance. Il cumule les fonctions de prêtre, guérisseur, exorciste, magicien, chef de chœur, organisateur de divertissements »(Hurbon, 1972, p. 90). Quant à elle, une mambo est une guérisseuse qui, comme un houngan, doit être possédée par un loa-guérisseur pour traiter des malades. Elle exploite les vertus thérapeutiques des plantes et utilise aussi des produits ethnopharmaceutiques.

Il faut noter que ce ne sont pas uniquement les matrones (sages-femmes traditionnelles), les médecins-feuilles, les houngans et les mambos qui réalisent des rites de stimulation de la production du lait et des rites de purification des accouchées. Ils peuvent être aussi accomplis par n'importe quelle personne qui s'y connaît en médecine créole : la grandmère, la mère, le père, une tante, une sœur, une voisine, une amie, etc.

Les données que nous avons recueillies nous permettent d'avancer que les rites d'allaitement se caractérisent par trois phases : la séparation, la marge et l'agrégation. Certaines accouchées se séparent de leur bébé en attendant que la production et la « descente »de leur lait soient induites par des gestes rituels spécifiques. Ensuite, durant l'allaitement, de nombreuses femmes s'interdisent non seulement de participer à certaines activités sociales, culturelles et économiques, mais aussi de toute relation amoureuse avec leur mari. Finalement, on constate la reprise de la sexualité et de certaines activités sociales, culturelles et économiques après le sevrage.

5. D'après le père d'une jeune paysanne allaitante, un loup-garou peut faire « partir » le lait d'une femme après l'avoir tétée : «Si un loup-garou ne peut pas tuer l'enfant qui vient de naître, il pourra téter sa mère durant son sommeil. Ce qui provoquera la disparition du lait maternel. » 
Les femmes allaitantes bénéficient des pratiques d'orientation du système environnemental. Par exemple, un membre de sa famille ou de sa communauté peut orienter une femme faisant face à une complication mammaire vers tel ou tel spécialiste de la médecine ancestrale. Le rôle des spécialistes de la médecine créole consiste à orienter les femmes allaitantes et à les aider à s'orienter lorsqu'elles rencontrent un problème de lait réel ou fantasmatique. On entend par orientation l'ensemble des processus physiologiques, cognitifs, mentaux, sociaux, culturels, institutionnels, affectifs, conscients, inconscients, etc., qui permettent à une personne de donner un sens à sa vie (auto-orientation) et à celle d'un autre (hétéro-orientation) sur le chemin de l'existence.

\section{La séparation (symbolique et réelle) d'avec le bébé, le mari et la communauté sociolinguistico-épistémique}

Beaucoup de femmes vivant en zone rurale ignorent l'existence du colostrum (premier lait) ainsi que le rôle de celui-ci dans la construction du système immunitaire de leur bébé. Elles ne mettent pas, durant les premiers jours, leur nouveau-né au sein sous prétexte que ce dernier ne contient pas encore de lait. L'ignorance de la valeur nutritionnelle et des propriétés purgatives du premier lait est à l'origine des actions rituelles ayant pour fonction de stimuler la production et la descente du lait maternel durant les deux jours suivant la naissance (la majorité des femmes allaitantes croient que le lait se fabrique dans la tête. Lorsqu'elles parlent de montée de lait, cela signifie que le lait remonte à la tête de la femme consécutivement à une émotion négative ou à un traumatisme). Cette ignorance explique le retard de l'émergence chez une accouchée du sentiment de continuité entre l'allaitement au sein et la vie intra-utérine de son bébé, sentiment dont l'absence serait la cause de la détresse psychique de certaines accouchées, d'après nos observations. On sait que l'allaitement établit un lien psycho-socio-affectif entre la mère et son enfant (fusion ou symbiose symbolique) et compense, dans une certaine mesure, la séparation, du point de vue physiologique, de celui-ci d'avec celle-là. Du point de vue symbolique, le sein se substitue au cordon ombilical ${ }^{6}$ qui, relié au placenta, assurait les échanges entre la mère et le fœtus dans le milieu intra-utérin. Il y a donc, dans une certaine mesure, un sentiment de continuité entre l'allaitement maternel et la vie intraplacentaire. Cependant, ce sentiment, dont elles ne sont pas conscientes, apparaît plus tard chez les femmes qui ne procèdent pas à l'allaitement au sein durant les deux ou les trois premiers jours qui suivent la naissance.

Chez les femmes qui font abstraction de la période colostrale, les rites de stimulation de la sécrétion lactée et de la descente du lait consistent essentiellement en massages et en bains de feuilles. On masse (façonne) les seins de la femme et on tamponne son corps avec des feuilles préalablement trempées dans de l'eau tiède ou chaude. Elle consomme certains aliments « galactogènes » (sorgho, papaye, igname blanc, fruit de l'arbre véritable, etc.) et en évite d'autres (tabous alimentaires: hareng salé, viande de porc...). Elle absorbe des tisanes, des infusions de feuilles ou des décoctions de plantes en vue de se purifier et de se blanchir le sang : «Le lait devient blanc après l'absorption des tisanes », raconte, une femme illettrée âgée de 75 ans, mère de treize enfants, dont huit sont morts. Les

6. Roman et Dublineau (2008) définissent le sein comme un cordon lacté : « ... l'allaitement viendrait alors soutenir une négation de la coupure du cordon ombilical et instaurer un cordon lacté bien plus fiable car davantage maîtrisable pour la jeune mère. » 
bains de feuilles sont aussi appelés bains de vapeur. Même les femmes qui ont recours à l'allaitement au sein immédiatement après la naissance de leur bébé bénéficient, d'une manière générale, de ces actions rituelles.

Christie (mère de trois enfants, couturière, revendeuse de produits alimentaires, 38 ans, a suivi une formation sur l'allaitement ${ }^{7}$ !) explique la fonction des massages et des bains de feuilles:

«On savonne les seins. On reçoit un bain de feuilles. On tamponne ton corps avec des feuilles pour que le lait puisse descendre. On masse les seins pour que les glandes se ramollissent. On jette l'eau du bain sur ton corps ». Camille (mère de deux enfants; 30 ans) raconte de son côté : « Le lait n'est pas descendu tout de suite. II est venu après deux ou trois jours. Quand on manque de lait, on te fait manger de la papaye. On te fait boire une décoction (de feuilles ou de racines?) de papayer pour que le lait descende. On a fait bouillir des feuilles. On m'a baignée. On a tamponné ma poitrine pour que le lait puisse descendre. Je me rappelle qu'on a utilisé des feuilles de "capable" de manguier, de corossolier et d'avocatier. »

La sage-femme traditionnelle Exantus Lajuce, que nous avons rencontrée à Jean-Rabel en 2012 lors d'une enquête auprès d'une trentaine de matrones, décrit le rituel du bain de vapeur en ces termes:

«Le bain de vapeur est mis dans un pot de nuit après la naissance du bébé. Il contient des feuilles. La chaleur des feuilles chaudes pénètre le vagin de l'accouchée. II ne faut pas que les feuilles soient trop chaudes, sinon on ferait du mal à cette dernière. II faut que l'eau du bain de feuilles soit tiède. On fait en sorte que la chaleur du bain de feuilles rentre dans le vagin de l'accouchée. Elle va faire fondre le sang. Si la chaleur est trop forte, on ne va pas atteindre le résultat escompté, car elle peut faire coaguler le sang. L'accouchée est alors malade. Elle reste dans son lit. Si on veut décoaguler le sang de manière progressive et faire revenir la cavité vaginale à son état normal, le bain de feuilles ne doit être ni chaud ni froid. Ensuite, on donne à la femme un autre bain de feuilles. Mais, on met les feuilles dans une cuvette d'eau. On presse ces feuilles sur son corps. Pendant qu'on lui donne le bain, on purge son ventre afin de le débarrasser du sang coagulé. Puis, on utilise des feuilles. Je fais toujours ceci : après lui avoir donné le bain de feuilles, je lui demande d'en boire l'eau [...] Je savonne son corps. Pour ce faire, j'utilise un savon de lessive. Ensuite, je lui lave le corps avec de l'eau traitée (j'utilise des Aquatabs). Cette dernière ne contient pas de feuilles. Si on laisse sur son corps de l'eau de feuilles, cela pourra lui donner de petits boutons. Les feuilles peuvent en être la cause. Après lui avoir donné le bain de feuilles, il faut nettoyer son corps. »

7. La formation sur l'allaitement n'a pas été assurée par les spécialistes de la médecine créole. Elle n'a pas empêché Christie de bénéficier des savoirs locaux sur l'allaitement maternel.

8. Nom vulgaire d'un arbre. 
Nombreuses sont les accouchées qui temporisent la première tétée ${ }^{9}$. Comme les femmes touarègues de l'Ahaggar (Barrère, 1987; Walentowitz, 2003) et mauritaniennes (Fortier, 2000), elles sont persuadées que leurs seins contiennent du mauvais lait, du lait immature ou dilué ( «lèt dlo »: lait non concentré ou dilué), donc impropre à la consommation néonatale. Par ailleurs, certaines d'entre elles pensent que le lait n'est autre chose que du sang, dont la transformation en lait (blanchiment) serait incomplète pendant les premiers jours après l'accouchement ( «C'est le sang de la mère qui s'est transformé en lait », raconte une femme multipare). Cette croyance représente l'un des obstacles à l'allaitement exclusif au sein pendant six mois. Elle se manifeste par la peur de devenir des sujets anémiques. Elle explique les conduites de prévention de l'agalactie complète (tarissement de la sécrétion lactée), du déficit de sécrétion lactée ou de l'hypogalactie (insuffisance de production lactée), lesquelles peuvent être observées dès la période de la grossesse. II s'agit de bien manger pour bien allaiter en sélectionnant des aliments 《lactogènes ». Pour Christie, il faut bien manger de crainte d'être anémiée par l'allaitement au sein : «Quand on allaite l'enfant au sein il faut bien se nourrir pour éviter le vertige. Je veux dire que c'est ton sang que l'enfant suce. Tu peux devenir anémique ». D'après une étude, il n'existe aucun lien entre la malnutrition des femmes allaitantes et la production lactée. Par exemple, on a découvert que les seins des femmes gambiennes sous-alimentées sécrétaient autant de lait que ceux des femmes anglaises bien alimentées (Prentice et al., 1986). L'enquête EMMUS-V (2013) nous rapporte : «Près d'une femme sur deux (49\%) est atteinte d'anémie : $37 \%$ sous la forme légère, $11 \%$ sous la forme modérée et $1 \%$ sous la forme sévère ». La peur d'être anémiées, dont les femmes allaitantes ne sont pas toujours conscientes, incite celles-ci à acheter des formules lactées (laits commerciaux, artificiels ou industriels) et à introduire précocement des compléments alimentaires liquides et solides. Elles pratiquent l'allaitement mixte (naturel et artificiel). Le sentiment qu'elles manquent de lait les pousse également à acheter des préparations commerciales. Elles interprètent mal les cris et les pleurs de l'enfant : un bébé qui pleure après avoir tété sa mère a besoin de suppléments alimentaires liquides ou solides : "Je dois donner à manger à un enfant qui pleure trop. Le lait maternel est insuffisant. L'enfant quitte le sein et se met à pleurer. Je suis obligé de lui donner à manger », raconte Victor, le mari de Guerda, une femme multipare. Quand l'enfant pleure, l'entourage familial de la femme lui demande de lui donner des aliments complémentaires. Le lait en poudre pour nourrissons demeure un aliment enviable aux yeux des mères extrêmement pauvres sur le plan économique : celles que j'ai rencontrées en 2016 à Jacmel et à Saint-Marc m'ont dit qu'elles achèteraient du lait en poudre pour nourrir leur bébé si elles avaient de l'argent ( $56 \%$ de la population haïtienne vit dans une pauvreté extrême). Un grand nombre de femmes allaitantes des milieux ruraux donnent du lait de vache à leur bébé à condition que celui-ci ait le même âge que le petit animal de la vache allaitante. Comme les femmes indiennes (Agnew et al., 1997), elles boivent du lait de vache afin de produire plus de lait.

9. La formation dont ont bénéficié quelques femmes de la part des acteurs formés à la médecine du Nord leur a permis d'allaiter leur bébé au sein immédiatement après leur naissance. Pour des raisons d'ordre culturel et historique, les femmes des milieux ruraux ne pratiquent pas l'allaitement exclusif au sein pendant six mois. Même les femmes plus ou moins cultivées et plus ou moins aisées des milieux urbains et ruraux ne le font pas pendant six mois de manière exclusive. II serait intéressant d'observer plusieurs femmes allaitantes qui ont suivi une formation sur l'allaitement. Mettent-elles toutes au sein leur bébé immédiatement après leur naissance? On sait que la tyrannie des mœurs empêche les gens de changer de comportement. Pour qu'une éducation à l'allaitement soit efficace, elle doit être fondée sur un pluralisme épistémologique. En d'autres termes, les spécialistes de la médecine créole doivent y être aussi impliqués. Dans ce cas, on peut parler de complémentarité entre l'épistémologie du Nord et les épistémologies du Sud en matière d'allaitement. 
Beaucoup d'accouchées consomment des aliments blancs (solides, liquides) afin de blanchir le lait de leurs seins. Ce sont des rites sympathiques. L'ethnologue Arnold Van Gennep (1981) les définit comme suit : «Sont rites sympathiques ceux qui se fondent sur la croyance à l'action du semblable sur le semblable, du contraire sur le contraire, du contenant sur le contenu et réciproquement, de la partie sur le tout et réciproquement, du simulacre sur l'objet ou l'être réel et réciproquement, de la parole sur l'acte. »La croyance au fait que le lait est du sang blanchi et cuit est aussi propre à l'Afrique ${ }^{10}$ dont les ancêtres des Haïtiens étaient originaires. On la rencontre particulièrement chez les Bambaras: "The Bambara believe that milk is produced'from the blood'and that each person has a finite amount of blood in her body for her lifetime. Therefore, it is not possible to increase or make-up any lost blood or affect the quantity of the milk through diet or medicine. If a woman loses a lot of blood in an accident, for example, she will not be able to produce much milk and may have to use formula to supplement breastfeeding. Older women who have already nursed many children will have a poor milk supply and will be tired all the time because they have'used up' all of their blood » (Katherine, 1987). Cette croyance était partagée par les médecins antiques, médiévaux et modernes qui « considéraient généralement que le lait n'est que le sang de la mère, un sang particulièrement pur et particulièrement "cuit". Lorsque la femme allaitait, ils pensaient que tout le "surplus" de son sang était transformé en lait au lieu d'être périodiquement évacué, et que cela expliquait la disparition des règles. [...]» (Knibiehler et Fouquet, 1977). Le médecin suisse, Albrecht Von Haller ${ }^{11}$, a développé dès 1757 une théorie selon laquelle le lait maternel se fabrique à partir de substances disponibles dans le sang (cette théorie a été évoquée lors d'une conférence prononcée en 2011 par Hartmann à l'occasion de la journée internationale de l'allaitement). La croyance que le lait vient de la cuisson du sang était également partagée par Aristote : « pendant la grossesse, l'enfant se nourrit du sang de la mère qui ne s'écoule plus; puis au moment de la naissance, le lait est produit à partir du sang menstruel, mais dans ce cas, « le lait est du sang qui a subi une coction parfaite et non du sang corrompu... Durant l'allaitement les règles n'ont pas lieu, si la nature suit son cours normal, et il n'y a pas de conception : si celle-ci se produit, le lait se tarit parce que la nature du lait est la même que celle des règles. Car la nature ne peut pas prodiguer ses efforts en deux directions à la fois : si la sécrétion se produit d'un côté, il est nécessaire qu'elle manque de l'autre, à moins qu'il ne s'agisse de quelque effet violent et contraire à la normale ${ }^{12}$. »

La séparation d'une femme allaitante d'avec son bébé et le jeûne infligé à celui-ci ne sont pas spécifiques à la culture haïtienne. En effet, ces pratiques étaient en vigueur dans les maternités françaises jusqu'en 1970. Dans sa thèse de doctorat en médecine, BorgnatJambon (2012) cite : «On imposait [...] une diète complète au nouveau-né pendant les deux premiers jours ce qui ne favorisait ni la montée laiteuse ni le réflexe de succion du bébé et les mères étaient séparées de leur enfant durant cette période ». Quantité de

10. Pour les Samo du Burkina Faso, les mécanismes de cuisson du sang existent aussi bien chez l'homme que chez la femme : "étant plus chaud que la femme, l'homme "cuit" le sang davantage, produisant ainsi une liqueur dense et éthérée (le sperme), tandis que la femme ne fabrique que du lait, qui est de même nature, mais moins dense et moins éthéré que le sperme » (Héritier-Augé, 1993).

11. Von Haller (1757-1766). Elementa physiologiae corporis humani, 8 tomes. Lausannae : Sumptibus M. M. Bousquet et Sociorum.

12. Aristote (1961). De la génération des animaux (texte établi et traduit par Pierre Louis). Paris : Les Belles Lettres. 
mères haïtiennes auraient pu faire l'économie de certains rites (les rites anti-agalactiques, antihypogalactiques ou hypergalactiques) si elles n'avaient pas recouru à ces pratiques culturelles (la séparation avec le bébé, l'imposition d'une diète à celui-ci). Nous pouvons émettre l'hypothèse qu'il y a une connexion entre ces dernières et le retard de la montée du lait. Hippocrate ${ }^{13}$ et Galien, médecins grecs de l'Antiquité, croyaient qu'il y avait une corrélation entre l'utérus et les seins, et que le sang cataménial s'orientait vers ces derniers pour nourrir le petit d'homme après la naissance (Hartmann, 2011). D'où, selon Hartmann, l'expression de la montée laiteuse qu'on utilise encore de nos jours. D'après les données collectées sur les croyances paysannes relatives au lait maternel, celui-ci ne doit pas, après la descente, remonter dans la tête d'une femme allaitante. Quand le « lait remonte à la tête » de cette dernière, cela peut la rendre folle, voire la tuer.

Les femmes primipares et multipares vivant en milieu rural que nous avons interviewées croient qu'il y a un lien entre la consommation de certains aliments et l'hypergalactie (production abondante de lait maternel). Les rites alimentaires anti-hypogalactiques ou hypergalactiques ont une dimension sympathique (rites sympathiques) et se fondent sur des principes de similarité et de contagion (Frazer, 1981). La croyance magico-alimentaire est liée au fait qu'on attribue aux aliments blancs ou blanchâtres le pouvoir de provoquer et d'augmenter la lactation tout en transmettant au lait leur couleur. « Le millet, l'igname blanche, l'arbre véritable, la papaye provoquent la surproduction de lait », raconte Rose (60 ans, multipare, mère de neuf enfants, dont trois sont morts).

Les rites alimentaires anti-hypogalactiques ou hypergalactiques sont dus à la peur de ne pas avoir assez de lait pour nourrir l'enfant. Par exemple, beaucoup de femmes brésiliennes utilisent le lait industriel, parce qu'elles ont le sentiment qu'elles n'ont pas assez de lait dans leur sein pour nourrir leur bébé (Rudzik, 2015). Dans les pays industrialisés, la perception d'insuffisance lactée est l'une des causes les plus fréquemment évoquées par les femmes pour mettre fin à l'allaitement et utiliser des formules commerciales (Galipeau, 2011 ; Ahluwalia et al., 2005; Li et al., 2008; Renfrew et al., 2005). Le syndrome d'insuffisance de lait aurait une dimension universelle.

\section{Le stade de la marge}

«Une femme qui se décide à nourrir doit former en même temps la résolution de renoncer aux bals, aux spectacles, aux grandes assemblées. Cette dissipation qui exalte les passions, est incompatible avec la vie sédentaire et paisible que demande l'allaitement ». Gardien (1812, p. 405)

À l'issue de l'accouchement, beaucoup de femmes restent dans leur cocon familial pour mieux allaiter leur enfant. Elles abandonnent provisoirement leurs activités sexuelles, commerciales et agraires. "Je faisais du commerce. Je ne fais rien parce que j'allaite mon enfant. Je ne peux pas sortir de chez moi. Le bébé est gâté. Je vendais mes produits dans la rue » raconte Yva (femme allaitante, 31 ans. Sa fillette est âgée de cinq mois). Camille vendait du savon de bain et de la crème de soin. Elle a dû arrêter ses activités économiques à cause de l'allaitement : «J'achetais et revendais du savon de bain et de la crème. Maintenant, je ne sors plus de chez moi ». La peur de ces femmes de redevenir

13. Hippocrate (1970). Génération. Paris : Les Belles Lettres. 
enceintes pendant l'allaitement au sein est la cause de l'abandon des rapports sexuels. Certaines mères pensent que si elles continuent d'allaiter, pendant une nouvelle grossesse, l'enfant pourra devenir une personne en situation de handicap. Bien qu'elles n'en soient pas conscientes, cette croyance multiséculaire leur permet d'espacer ou de réguler les naissances. Soranos d'Éphèse, médecin grec du début du $2^{\mathrm{e}}$ siècle après Jésus-Christ, a écrit dans un livre intitulé «Maladies des femmes »: «La copulation gâte le lait et le tarit partiellement ou totalement en réveillant le flux menstruel de la matrice et en aboutissant à la conception » (1990). La croyance selon laquelle il est dangereux d'allaiter un enfant dès qu'on sait qu'on fait face à une nouvelle gestation est largement partagée par les femmes haïtiennes, quels que soient leur niveau d'éducation et leur origine sociale. D'après Rose (une femme multipare), les femmes qui tombent enceintes pendant qu'elles allaitent un bébé doivent arrêter l'allaitement ou continuer à l'allaiter avec un seul sein : « II y a des femmes, dit-elle, qui continuent à allaiter l'enfant avec un seul sein. Moi-même, je cesse de lui donner mon sein ». Donner un seul sein à un bébé lorsqu'on est enceinte de nouveau est un geste symbolique. II s'agirait de ne pas susciter la jalousie de l'être qui se développe dans le corps maternel, être auquel on attribue un psychisme.

La durée de l'allaitement au sein varie avec le niveau d'éducation et le statut socioéconomique des mères. Chez celles qui sont illettrées, l'allaitement peut durer jusqu'à 24 mois $^{14}$. Ce sont les femmes éduquées des milieux ruraux et urbains qui transgressent souvent la norme universelle que constitue l'allaitement au sein. Selon une étude réalisée par Grummer-Strawn (1996), dans les pays en voie de développement, les nouveau-nés des femmes analphabètes ont 1.9 fois plus de chance d'être nourris au sein que ceux dont la mère a bénéficié de sept ans d'études. D'après les faits que nous avons recueillis sur l'allaitement en Haïti, ce sont les femmes qui savent lire qui écourtent le plus souvent la période de marge. En voici un exemple : Josie (36 ans) est arrivée en classe de première. Elle est institutrice. Elle vit à la campagne. Comme elle a suivi une formation sur l'allaitement, elle connaît les bénéfices pour son bébé de l'allaitement exclusif au sein pendant six mois. En outre, elle sait que l'allaitement la protège contre les cancers du sein, du col de l'utérus et des ovaires. À cause de son travail, elle a allaité son fils de cinq mois au sein durant deux mois et demi uniquement. Elle manque de lait puisqu'elle n'est pas disponible pour son bébé (rapport entre la fréquence des tétées et la lactation). Celui-ci sera sevré quand il marchera. Donc, elle ne pratiquera pas l'allaitement prolongé.

Dans les pays industrialisés, contrairement aux femmes pauvres, les mères instruites allaitent leur enfant au sein de manière exclusive pendant six mois (Galipeau, 2011 ; Thulier et Mercer, 2010; Wambach et al., 2005; Renfrew et al., 2005; Dennis, 2002). L'allaitement prolongé et le maternage proximal (attachment parenting) qui contribuent à la santé physique et psychique de la mère et de l'enfant (Bartick et al., 2013; Turck et al., 2013 ; Goldman et al., 1983) sont des pratiques marginales dans ces pays, alors qu'ils sont des normes dans de nombreuses sociétés traditionnelles et paysannes.

L'allaitement au sein est un apprentissage. Les femmes rurales primipares apprennent des règles et des normes reliées à l'allaitement maternel pendant la période de marge. Ces règles et ces normes se transmettent de mère en fille ou de grand-mère en petite-fille. Les

14. Mais les rapports sexuels ne sont pas interdits durant toute cette période. Chez les Samo du Burkina Faso, la sexualité est interdite pendant les deux ans que dure l'allaitement (Héritier-Augé, 1993). 
femmes primipares apprennent les rites de préparation à l'allaitement (lavage et massage des seins, expression du lait, stimulation des mamelons, lavage des mains avant de prendre le sein pour allaiter l'enfant) et d'autres règles comme la maîtrise des émotions négatives (éducation émotionnelle), l'allaitement sur demande, le fait de ne pas allaiter quand on a chaud (on pense que le lait « chaud»du sein peut provoquer la diarrhée chez le bébé), le fait de bien se nourrir pour bien allaiter son enfant, etc. Les mères apprennent à leur fille les positions à adopter pour allaiter. En voici un exemple :

« Je lui fais prendre des précautions ${ }^{15}$ quand elle allaite l'enfant. Elle doit tenir son sein de telle manière qu'il ne puisse élargir la bouche du bébé. Lorsqu'elle se couche, elle ne doit pas mettre le sein dans la bouche de ce dernier pour la raison suivante: puisque l'enfant est petit, cette position peut l'empêcher de respirer. De plus, si la mère s'endort, le sein peut boucher le nez de l'enfant » (Conseils de Jocelyne, mère de sept enfants dont deux sont morts, à sa fille Rita, une femme allaitante).

Dans la plupart des sociétés, le congé de maternité permet aux femmes qui vont accoucher de rester chez elles, pendant une certaine période, pour allaiter leur bébé. En Haïti, d'après l'article 321 du Code du travail, le congé de maternité dure trois mois. Mais la rareté des ressources humaines de qualité et le pouvoir discrétionnaire de certains patrons constituent des obstacles à l'octroi de ce congé aux femmes enceintes qui en font la demande. Si les femmes salariées et plus ou moins cultivées, qui vivent dans les campagnes et les villes haïtiennes, passent moins de temps à la maison pour nourrir au sein leur enfant, c'est parce qu'il leur est difficile d'obtenir un congé de maternité auprès de leur patron. Les femmes rurales, dont l'économie est agro-pastorale ou agrosylvo-pastorale, sont plus indépendantes du point de vue financier que celles des milieux urbains. Cependant, plus une femme allaitante résidant en milieu rural est pauvre, plus elle est obligée de raccourcir la période de marge. La durée de cette dernière dépend aussi de la situation socio-économique du père de l'enfant.

On continue d'avoir recours à des rites alimentaires et à des recettes galactogènes afin d'avoir suffisamment de lait pour nourrir l'enfant durant la période de marge. Si une femme perd son lait à la suite d'un choc émotionnel, des bains de feuilles et des massages seront pratiqués afin d'induire le retour du processus de lactation.

Pauline nous raconte:

« Un traumatisme peut bloquer la production de lait. La lactation se tarit. On prend des "médicaments" pour faciliter la descente du lait dans le sein. On a jeté de l'eau d'un bain de feuilles sur ma tête. On a utilisé des feuilles lactogènes, de la cassave et du hareng saur. Je ne me souviens pas de toutes les feuilles. Parfois, on en a fait bouillir; on a laissé le bain de feuilles au soleil. On y a ajouté des feuilles de caféier et des feuilles qui favorisent le

15. Note du traducteur : L'une des précautions à prendre par une femme allaitante vivant en milieu rural haïtien consiste à éviter que le pénis de l'enfant n'entre en contact avec le lait maternel. Si une goutte de lait tombe sur son pénis, celui-ci ne pourra jamais entrer en érection. Chez les Wolofs du Sénégal, le contact des organes sexuels du bébé avec le lait de la mère fera de lui une personne volage (Ndiaye, 2018). 
retour du lait maternel (fèy lèt manman). On m'a massée avec toutes les feuilles. Finalement, le lait est descendu dans les seins. »

Aussi paradoxal que cela puisse paraître, la pauvreté financière est un facteur qui favorise l'allaitement maternel. «Nous n'avons pas d'argent pour acheter du lait en poudre. Nous sommes obligées de leur donner nos seins. Je ne donnais pas de lait commercial à mes enfants », raconte Jocelyne.

\section{La période d'intégration}

La période de réintégration se caractérise par la reprise des activités commerciales, agricoles et sexuelles. Le début de la réintégration est relié à une constellation multifactorielle : l'âge de la mère, le nombre d'enfants de la famille, le statut socio-économique, le niveau d'éducation de la mère et du père, etc. II coïncide avec les rites de sevrage qui se réalisent lorsque l'enfant peut marcher sans difficulté. Les bouts de sein de la mère sont enduits d'un liquide amer extrait d'une plante médicinale appelée aloe vera : « J'ai passé du jus d'aloe vera sur les seins pour dégoûter l'enfant », dit Camille. Désormais, l'enfant est perçu comme un être plus ou moins autonome (« il marche, il court, il devient indépendant », dit une mère), tandis qu'on cédait à ses caprices durant la période de marge. Les rites de sevrage sont des rites d'intégration qui consistent à « introduire l'enfant dans le monde »(Van Gennep, 1981). La mère peut se séparer de son enfant en l'envoyant chez sa mère, sa grand'mère, sa tante ou chez une amie. La mère en profite pour régler certaines activités personnelles et familiales. L'enfant sevré peut passer quelques jours ou des mois chez la personne qui l'a tenu sur les fonts baptismaux (son parrain ou sa marraine) ou être adopté provisoirement ou définitivement par une personne (Kou I sevre, ou mèt vin pran I: Tu viendras le chercher quand il sera sevré). Dès lors qu'il est sevré, il est capable de passer beaucoup plus de temps en dehors de la cellule familiale. Le processus de sa socialisation extrafamiliale s'intensifie.

\section{Conclusion}

Comme le montre cette étude, l'allaitement des femmes haïtiennes est ancré dans un réseau labyrinthique de représentations individuelles et collectives. II est une construction sociale et culturelle. II participe à la construction de l'enfant tant sur le plan physiologique que social. Les femmes allaitantes peuvent se tourner vers leur mère, leur sœur, leur voisine, leur amie, les matrones (épistémologies féministes), les houngans (guérisseurs), les mambos (guérisseuses), les médecins-feuilles, la providence divine, etc., selon les attributions causales du problème de lait réel ou imaginaire auxquelles elles font face. La réalité de l'allaitement humain dans les communautés rurales haïtiennes est construite par des croyances, des rationalités et des représentations qui guident les stratégies de recherche d'aide des femmes qui éprouvent des problèmes de lait, problèmes dont la prise en charge relève non seulement de l'entraide féminine, mais encore de la compétence de certains hommes communément appelés les docteurs-feuilles et les houngans. Le rôle de ces tradipraticiens consiste justement à renforcer le sentiment de compétence des mères (Lent et al., 1994 ; Bandura, 1986, 1977), c'est-à-dire leur confiance dans la capacité d'allaiter leur enfant, lorsqu'elles doivent affronter le syndrome d'insuffisance lactée ou lorsqu'elles pensent que leur lait est « troublé, contrarié, tourné, dilué », etc. 
Les faits collectés nous permettent d'avancer que l'acquisition du statut de mère, dans les campagnes haïtiennes, semble être intimement liée au rôle nourricier d'une femme allaitante. Ainsi, l'allaitement au sein permet à une femme de passer dans le monde des mères. Le rituel d'allaitement est constitutif de son identité féminine et maternelle : une bonne mère est une personne qui allaite son enfant. Une femme qui déroge à cette règle sera considérée comme une personne méchante (《un loup-garou ») par les membres de sa communauté. Elle ne doit pas essayer d'échapper au déterminisme biologique de l'allaitement. L'intérêt que l'entourage familial et communautaire porte à la vie d'une femme et de son enfant est tel qu'il est difficile à celle-là de prendre la décision de ne pas allaiter celui-ci. L'allaitement serait donc une norme non négociable au sein de la communauté morale. Sa réussite dépend d'une harmonisation entre les deux systèmes moosiens (Moos, 1987) : le système environnemental (familles, communauté, voisinage, église, etc.) et le système personnel (personnalité, caractéristiques socio-démographiques, compétence, préférences). On peut noter à juste titre que si cette norme que constitue l'allaitement au sein n'était pas respectée, la pauvreté économique des familles rurales augmenterait. En Occident, l'absence d'allaitement est un facteur d'appauvrissement des familles les plus vulnérables (Turck, 2010).

Quant à l'enfant, l'allaitement lui permet de passer d'une dépendance totale à une autonomie relative grâce au rite de sevrage. Sa mère peut passer plus de temps à l'extérieur de la maison, notamment dans les champs, le voisinage, les marchés locaux... Le sevrage peut se définir comme la rupture du cordon ombilical symbolique ou psychique qui reliait, après la naissance, la mère à l'enfant. Le processus de socialisation de ce dernier se renforce grâce à la participation d'autres catégories sociales, notamment les grands-parents paternels et maternels, à sa prise en charge et à son éducation.

La diversité des savoirs du Sud reliés à l'allaitement (perspective compréhensive) témoigne, d'une part, de l'incomplétude des savoirs dits scientifiques prétendant expliquer ce phénomène et, de la lutte consciente et inconsciente des communautés (qualifiées à tort d'ignorantes par les détenteurs de savoirs universels) contre les pratiques exo et endoépistémicides ${ }^{16}$, d'autre part. En somme, le dynamisme de ces savoirs alternatifs indique que les communautés « idiotes culturelles» luttent avec efficacité contre la tendance des sciences dominantes à détruire les savoirs dits profanes sur l'allaitement (il s'agit d'un processus scientophagique à la fois conscient et inconscient). Contrairement aux savoirs dits scientifiques, les savoirs dits profanes sur l'allaitement maternel sont accessibles à toutes les couches sociales en Haïti. Au nom du double principe d'équité inter et intra-générationnelle et au nom de la justice cognitive (Santos, 2016), il est urgent de transmettre et de sauvegarder ces savoirs alternatifs sur l'allaitement maternel (pédagogie émancipatrice).

Plusieurs raisons expliquent la non-remise en question de l'allaitement maternel dans les communautés rurales : a) la disponibilité des mères, b) la pauvreté économique, c) l'analphabétisme ou le faible niveau d'éducation, d) le sentiment religieux, e) la croyance à la

16. On peut appeler exo-épistémicide la destruction des expertises locales par les interventions occidentalocentrées dans le domaine de l'allaitement. Après avoir suivi une formation eurocentrée ou américanocentrée, les femmes peuvent rejeter un certain nombre de savoirs locaux sur l'allaitement, qui étaient jusque-là efficaces, même s'ils ne correspondaient pas au canon épistémologique de la Science occidentale. Il s'agit, dans ce cas, d'un endoépistémicide qui s'explique par la force aliénatrice de l'épistémè eurocentré ou américano-centré en éducation à l'allaitement (pédagogie des dominants). 
supériorité du lait maternel (« lèt manman se lavi » : le lait maternel est un aliment vital) par rapport aux laits commerciaux, f) la croyance selon laquelle les enfants bien allaités seront forts physiquement, g) le respect inconditionnel des caprices des bébés avant la marche, h) le fait que les seins ne sont pas perçus comme des organes érotiques (les seins sont des organes nourriciers qui appartiennent à l'enfant, à la mère et non au père), i) le fait que les femmes rurales ne sont pas exposées à la publicité télévisée de l'industrie alimentaire infantile à la différence de nombreuses femmes urbaines et occidentales (la commercialisation des formules lactées nourrit chez les femmes occidentales le sentiment d'insuffisance lactée), j) la coopération entre les mères rurales ${ }^{17}$, etc. Par ailleurs, l'allaitement au sein confère à la mère un pouvoir magico-symbolique sur son enfant. En effet, certaines mères paysannes haïtiennes pensent qu'elles puniront un enfant qui ne les respecte pas si elles donnent au soleil leur sein gauche (magie du geste).

Malgré sa valeur heuristique et méthodologique, le schéma van gennepien ne nous a pas permis de rendre totalement justice à la complexité des faits relatifs aux représentations sociales et culturelles de l'allaitement en milieu rural haïtien. Notre interprétation de l'allaitement maternel en termes de rite de passage est relative dans la mesure où les rites de séparation, de marge et de réintégration qui le caractérisent ne sont pas absolument cloisonnés dans la réalité empirique. Par exemple, la période de marge se caractérise non seulement par la rupture de la femme allaitante avec des activités commerciales et sexuelles, mais encore par le renforcement du lien vicinaire (solidarité des voisines), amical, religieux (solidarité des membres de son église, dont les femmes), intragénérationnel, intergénérationnel, sororal (solidarité des sœurs biologiques et des coreligionnaires féminines), etc. La femme allaitante n'est pas complètement séparée de sa communauté sociolinguisticoépistémique. Bien au contraire, cette dernière l'oriente et l'accompagne dans le processus d'allaitement. Les invariants représentationnels de l'allaitement ne doivent pas nous faire oublier que nos analyses se fondent sur un échantillon restreint de femmes allaitantes. L'allaitement humain est l'objet de représentations masculines et féminines contrastées qui varient d'une communauté rurale à l'autre. Notre démarche d'analyse relève donc du relativisme épistémologique. II n'en reste pas moins que notre article constitue une modeste contribution au renouvellement de l'analyse classique de l'allaitement comme rite de passage.

\section{Références bibliographiques}

Ahluwalia, I.B., Morrow, B. et Hsia, J. (2005). Why Do Women stop breastfeeding? Findings from the Pregnancy Risk Assessment and Monitoring System. Pediatrics, 116, 1408-1412.

Agnew, T., Gilmore, J. et Sullivan, P. (1997). Perspective multiculturelle de l'allaitement maternel au Canada. Ministère des travaux publics et Services gouvernements.

Aristote (1961). De la génération des animaux (P. Louis, Trad.). Paris : Les Belles Lettres.

Badinter, É. (2010). Le conflit, la femme et la mère. Paris : Flammarion.

Bardin, L. (1993). L'analyse de contenu. Paris : PUF.

Bartoli, L. (1998). Venir au monde. Les rites de l'enfantement sur les cinq continents. Paris : Payot.

17. D'après les témoignages collectés, quand une femme allaitante s'absente, son bébé peut être nourri gratuitement au sein par une autre maman (sœur, voisine, amie, etc.). Une jeune femme peut confier l'allaitement au sein de son enfant à une femme de son âge ou à une autre femme plus âgée. Actuellement, la peur des maladies infectieuses pousse certaines mères à remettre en question cette pratique de solidarité humaniste ou allopoïétique. 
Bartick, MC., Stuebe, AM., Schwarz EB., Luongo, C., Reinhold, AG. Et Foster, EM. (2013). Cost analysis of maternal disease associated with suboptimal breastfeeding. Obstetrics \& Gynecology, 122(1), 111-119.

Barrère, G. (1987). Naissance et baptême des enfants à Idélès (Ahaggar). Université de Provence, Travaux du LAPMO.

Bandura, A. (1986). Social foundations of thought and action : a social cognitive theory. Englewoods Cliffs, NJ, Prentice-Hall.

Bandura, A. (1977). Social learning theory. Englewoods Cliffs, NJ, Prentice-Hall.

Bitoun, P. (1994). Valeur économique de l'allaitement maternel. Les Dossiers de l'obstétrique, 216, 10-13.

Borgnat-Jambon, A. (2012). L'allaitement maternel en médecine générale : Représentations, attitudes pratiques des médecins généralistes et perception du vécu de leurs patientes. Étude qualitative auprès de 17 médecins généralistes de Rhône-Alpes. Thèse de doctorat non publiée, Université Claude Bernard Lyon 1.

Damus, O. (2019). Solidarité et handicap en Haïti. [Solidarity and disability in Haiti]. Alternativas. Cuadernos de Trabajo Social, 26 (p. 83-102). https ://doi.org/10.14198/ALTERN2019.26.04

Damus, O. (2017). Las prácticas profesionales de las comadronas tradicionales en Haití : entre salud, biodiversidad y espiritualidad_. In B. Baptiste et al (Eds), Knowing_our Lands and Resources. Indigenous and Local Knowledge of Biodiversity and Ecosystem Services in the Americas (p. 20-31). Paris : UNESCO.

Damus, O. (2016). Homo vulnerabilis. Repenser la condition humaine (préface de Monique Castillo). Paris : Publibook.

Damus, O. (2015). La pensée, la connaissance et l'action rituelle des sages-femmes traditionnelles d'Haïti. In D. Jeffrey (Ed), La fabrication des rites (pp. 89-109). Québec : PUL.

Damus, O. (2012). Les rites de naissance en Haïti. Paris : L'Harmattan.

Dasen, V. (2015). Le sourire d'Omphale. Maternité et petite enfance dans l'antiquité. Rennes : Presses universitaires de Rennes.

Del Valle, M. B. (2000). La naissance, un voyage. L'accouchement à travers les peuples. Paris : L'Harmattan.

Dennis, C.-L. (2002). Breastfeeding initiation and duration : a 1990-2000 literature review. Journal of Obstetric, Gynecologic and Neonatal Nursing, 31(1), 12-32.

Desclaux, A. et Taverne, B. (2000). Allaitement et VIH en Afrique de l'Ouest. De l'anthropologie à la santé publique. Paris : Karthala.

Erny, P. (1988). Les premiers pas dans la vie de l'enfant d'Afrique noire. Naissance et première enfance. Paris : L'Harmattan.

Erny, P. (1994). La notion de rite de passage. In TG. D'Allondas (Ed), Rites de passage : d'ailleurs, ici, pour ailleurs. Érès : Ramonville-Saint-Agne

Frazer, J. (1981). Le rameau d'or. Paris : Robert Laffont.

Fortier, C. (2000). Corps, différence des sexes et infortune : transmission de l'identité et des savoirs en islam malékite et dans la société maure de Mauritanie. Thèse de doctorat (sous la direction de Pierre Bonte). Paris : EHESS.

Galipeau, R. (2011). Modélisations de facteurs associés à une perception d'insuffisance lactée. Thèse présentée à la Faculté des sciences infirmières en vue de l'obtention du grade de Ph.D. en sciences infirmières. Thèse non publiée, Université de Montréal.

Gardien (1812). Allaitement in Dictionnaire des sciences médicales. Paris : Panckoucke.

Grummer-Strawn, LM. (1996). The effect of changes in population characteristics on breastfeeding trends in 15 developing-countries. Int J of Epidemiol ; 25(1), 94-102.

Goldman, AS., Goldblum, RM. et Garza, C. (1983). Immunologic components in human milk during the second year of lactation. Acta Paediatrica, 72(3), 461-462.

Hartmann, P. (2011). Le rite de passage du lait humain. De la synthèse dans le sein maternel à l'estomac du bébé. Conférence prononcée par le professeur Hartmann lors de la journée internationale de l'allaitement 2011.

Héritier-Augé, F. (1993). Une anthropologie symbolique du corps. In S. Arom et al. (Eds), La Science sauvage. Des savoirs populaires aux ethnosciences. Paris : Seuil.

Hippocrate (1970). Génération. Paris : Les Belles Lettres.

Hurbon, L. (1972). Dieu dans le vaudou haïtien Paris : Payot. 
Katherine, DA. (1987). Breastfeeding and weaning in Mali. In Cultural context and hard data in Social Science and Medicine, 24(8), 633-644.

Knibiehler, Y. et Fouquet, C. (1980). L'Histoire des mères du Moyen Âge à nos jours. Paris : Montalba.

Lett, D. et Morel, M.-F. (2006). Une histoire de l'allaitement. Paris : La Martinière.

Li, R., Fein, SB., Chen, J., et Grummer-Strawn, LM. (2008). Why Mothers Stop Breastfeeding : Mothers' Selfreported Reasons for Stopping During the First Year. Pediatrics, 122, S69-S76.

Lent, RW., Brown, SD. et Hackett, G. (1994). "Towards a Unifying Social Cognitive Theory of Career and Academic Interest, Choice and Performance", Journal of Vocational Behavior, 45, 79-122.

Moos, R. H. (1987). Person-environment Congruence in Work, School, and Health Care Setting. Journal of Vocational Behavior, 31, 231-247.

Prentice, A., et al. (1986). Cross-cultural differences in lactational performance. In M. Hamosh, et AS. Goldman (Eds), Human Lactation 2, Maternal and Environmental Factors (p. 13-44). New York : Plenum Press.

Pourchez, L. (2011). Savoirs des femmes, médecine traditionnelle et nature. Maurice, Réunion, Rodrigues. Paris : UNESCO.

Radford, A. (1992). The ecological impact of bottle feeding. Breastfeeding review, 2(5), 204-2008.

Renfrew, M., Dyson, L., Wallace, L., D'souza, L., Mccormick, F. et Spib, H. (2005). The effectiveness of public health interventions to promote the duration of breastfeeding : a systematic review. London : National Institute for Health and Clinical Excellence.

Rollet, C. (2005). Histoire de l'allaitement en France : pratiques et représentations. Université de Versailles SaintQuentin-en-Yvelines, Laboratoire Printemps CNRS.

Roman, P. et Dublineau, M. (2008). Allaitement maternel prolongé militant et constitution du lien générationnel. Apport de l'épreuve du Rorschach. Le Divan familial ( $N^{\circ} 20$ ), p. 151-169. DOI 10.3917/difa.020.0151.

Rudzik, A. E. F. (2015). «The embodied experience of breastfeeding and the product/process dichotomy in São Paulo, Brazil » in CASSIDY T. et EL TOM A. (éds.) Ethnographies of Breastfeeding : Cultural Contexts and Confrontations. London : Bloomsbury Academic.

Santos, B. de S. (2016). Épistémologies du Sud. Mouvements citoyens et polémiques sur la science. Paris : Desclée De Brouwer.

Soranos D'Éphèse (1990). Maladies des femmes. Tome II. Livre II (texte traduit et commenté par Burguiere Paul, Gourevitch Danielle et Malinas Yves). Paris : Les Belles Lettres.

Thirion, M. (2010). Histoire de l'allaitement. in Frydman, R. et Szejer, M. (Eds), La naissance : histoire, cultures et pratiques d'aujourd'hui. Paris : Albin Michel.

Thulier, D. et Mercer, J. (2010). Variables associated with breastfeeding duration. Journal of Obstetric, Gynecologic and Neonatal Nursing, 38, 259-268.

Turck, D. (2010). Propositions d'actions pour la promotion de l'allaitement maternel, « Plan d'action : Allaitement maternel ». Lien de téléchargement du rapport : http://solidaritessante.gouv.fr/IMG/pdf/Rapport_Plan_daction allaitement_Pr_D_Turc_pdf

Turck, D. et al. (2013). Allaitement maternel, les bénéfices pour la santé de la mère et de l'enfant. https://www. sciencedirect.com/journal/archives-de-pediatrie/vol/20/suppl/S2

Van Gennep, A. (1981, [1909]). Les rites de passage. Paris : A. et J. Picard.

Walentowitz, S. (2003). «Enfant de Soi, enfant de l'Autre ». La construction symbolique et sociale des identités à travers une étude anthropologique de la naissance chez les Touaregs (Kel Eghlal et Ayttawari de l'Azawagh, Niger). Thèse de doctorat non publiée, École des Hautes Études en Sciences Sociales.

Wambach, K., Campbell, S., Gill, S., Dodgson, J., Abiona, T. et Heinig, M. (2005). Clinical lactation practice : 20 years of evidence. Journal of Human Lactation, 21(3), 245-258. 